九州大学学術情報リポジトリ

Kyushu University Institutional Repository

\title{
Extracting Best Consensus Motifs from Positive and Negative Examples
}

Tateishi, Erika

Department of Information Systems, Kyushu University

Maruyama, 0samu

Department of Information Systems, Kyushu University

Miyano, Satoru

Research Institute of Fundamental Information Science Kyushu University

http://hdl. handle. net/2324/3217

出版情報 : RIFIS Technical Report. 115，1995-06-08. 九州大学理学部附属基礎情報学研究施設 バージョン：

権利関係 : 


\title{
RIFIS Technical Report
}

\author{
Extracting Best Consensus Motifs \\ from Positive and Negative Examples
}

Erika Tateishi

Osamu Maruyama

Satoru Miyano

June 8, 1995
Revised: August 21, 1995

Research Institute of Fundamental Information Science

Kyushu University 33

Fukuoka 812, Japan

E-mail: tateishi@rifis.kyushu-u.ac.jp

Phone: 092-641-1101 ex. 4459 


\title{
Extracting Best Consensus Motifs from Positive and Negative Examples
}

\author{
Erika Tateishi, Osamu Maruyama*and Satoru Miyano ${ }^{\dagger}$ \\ ${ }^{*}$ Department of Information Systems, Kyushu University 39, Kasuga 816, Japan \\ ${ }^{\dagger}$ Research Institute of Fundamental Information Science \\ Kyushu University 33, Fukuoka 812, Japan
}

\begin{abstract}
We define the best consensus motif (BCM) problem motivated by the problem of extracting motifs from nucleic acid and amino acid sequences. A type over an alphabet $\Sigma$ is a family $\Omega$ of subsets of $\Sigma^{*}$. A motif $\pi$ of type $\Omega$ is a string $\pi=\pi_{1} \cdots \pi_{n}$ of motif components, each of which stands for an element in $\Omega$. The BCM problem for $\Omega$ is, given a yes-no sample $S=\left\{\left(\alpha^{(1)}, \beta^{(1)}\right), \ldots,\left(\alpha^{(m)}, \beta^{(m)}\right)\right\}$ of pairs of strings in $\Sigma^{*}$ with $\alpha^{(i)} \neq \beta^{(i)}$ for $1 \leq i \leq m$, to find a motif $\pi$ of type $\Omega$ that maximizes the number of good pairs in $S$, where $\left(\alpha^{(i)}, \beta^{(i)}\right)$ is good for $\pi$ if $\pi$ accepts $\alpha^{(i)}$ and rejects $\beta^{(i)}$. We prove that the BCM problem is NP-complete even for a very simple type $\Omega_{1}=\{z \mid \emptyset \neq z \subseteq \Sigma\}$, which is used, in practice, for describing protein motifs in the PROSITE database. We also show that the NP-completeness of the problem does not change for the type $\Omega_{\infty}=$ $\Omega_{1} \cup\left\{\Sigma^{+}\right\} \cup\left\{\Sigma^{[i, j]} \mid 1 \leq i \leq j\right\}$, where $\Sigma^{[i, j]}$ is the set of strings over $\Sigma$ of length between $i$ and $j$. Furthermore, for the BCM problem for $\Omega_{1}$, we provide a polynomial-time greedy algorithm based on the probabilistic method. Its performance analysis shows an explicit approximation ratio of the algorithm.

Keywords: algorithms and computational complexity, genome informatics
\end{abstract}

\section{Introduction}

PROSITE Database [3] compiles various important "motifs" of amino acid sequences of proteins. Most motifs have been extracted by biological experiments and alignment techniques tuned up with knowledge on molecular biology. Such motifs are expressed in a systematic way in PROSITE. For example, a motif of some family of DNA-binding proteins is expressed as a pattern W- $x(2)$-[LI]-[SAG]- $x(4,5)-\mathrm{R}-x(8)-[\mathrm{YW}]-x(3)$-[LIVM]. Here each component in the motif is separated from its neighbor by '-'. The component of the form [symbols] represents that any of the symbols between the brakets is accepted for the position. For example [SAG] stands for "S or A or G." The component $x(i, j)(x(l))$ represents any sequences of length between $i$ and $j$ (of length $l$ ). Another example of a motif is $[\mathrm{AC}]-x-\mathrm{V}-x(4)-\{\mathrm{ED}\}$, where $x$ is used for a position where any symbol is accepted and $\{E D\}$ stands for any symbol except $E$ and $D$. Thus this can be translated as [A or C]-any-V-any-any-any-any-\{any but E or D $\}$.

Finding such motifs from sequence data is a very crucial problem in Genome Informatics/Molecular Bioinformatics since motifs provide us biologically important knowledge in terms of amino acid residues. Traditionally, various alignment techniques [5], which shall find a "reasonable" common subsequence for a family of amino acid sequences or nucleic acid sequences, have played a significant role in discovering these motifs from sequence data.

In this paper we consider the problem of finding such "motifs" from a collection of positive and negative examples while alignment techniques deal with only positive examples. We call a string $\pi=\pi_{1} \cdots \pi_{n}$ a motif, where $\pi_{1}, \ldots, \pi_{n}$ stand for such components as V, [SAG], \{ED\}, $x(i, j), x(l)$ and $x$. A yes-no sample is a set $S$ of pairs $\left(\alpha^{(1)}, \beta^{(1)}\right), \ldots,\left(\alpha^{(m)}, \beta^{(m)}\right)$ of positive

\footnotetext{
${ }^{1}$ Corresponding author: Erika Tateishi, Research Institute of Fundamental Information Science, Kyushu University 33, Fukuoka 812, Japan. Email: tateishi@rifis.kyushu-u.ac.jp.
} 
and negative examples. A pair $\left(\alpha^{(i)}, \beta^{(i)}\right)$ in $S$ is said to be good for a motif $\pi$ if $\pi$ accepts $\alpha^{(i)}$ and rejects $\beta^{(i)}$. The best consensus motif (BCM) problem is, given a yes-no sample $S$, to find a motif that maximizes the number of good pairs in $S$. Some interesting results have been shown in $[6,7]$ for the consistency problem of regular patterns [1] from the point of computational learning theory. But regular patterns do not allow ambiguity in their expressions. An important motivation for defining the BCM problem is that sequences in the biological databases such as PIR, PDB, GenBank, etc., involve diversity and uncertainty.

The problem of finding, if any, a motif $\pi$ such that all yes-no examples in $S$ are good for $\pi$ can be solved in polynomial time if the motif is restricted to use only components of the form [symbols]. In contrast, we show that the BCM problem for this special case turns NP-complete as in the case of MAX2SAT [4]. More generally, the BCM problem for motifs with [symbols], $x(i, j), x(l), x$ and $x^{+}$is also shown NP-complete, where $x^{+}$represents the set of all nonempty strings.

For such obstacles to knowledge discovery from sequences, a big challenge is to devise efficient heuristic algorithms for approximating the BCM problem. We do not know whether it is possible to obtain any polynomial-time algorithm with any explicit performance guarantee. This paper answers a special case of the problem by showing a simple greedy algorithm approximating the BCM problem for motifs with components [symbols]. We prove a lower bound of its performance by a rigorous analysis of the algorithm with a probabilistic method in [14].

This paper is organized as follows: In Section 2, we define the best consensus motif problem. The NP-completeness of the problem is proved in Section 3. In Section 4, we give a greedy algorithm for the special case of the BCM problem and its performance analysis.

\section{Best Consensus Motif Problem}

For an alphabet $\Delta$, we denote by $\Delta^{*}$ the set of all strings over $\Delta$. The length of a string $w$ in $\Delta^{*}$ is denoted by $|w|$. We denote $\Delta^{+}=\Delta^{*}-\{\varepsilon\}$ ( $\varepsilon$ is the empty string). For $1 \leq l$ and $1 \leq i<j$, let $\Delta^{l}=\left\{w \in \Delta^{*}|| w \mid=l\right\}$ and $\Delta^{[i, j]}=\left\{w \in \Delta^{*}|i \leq| w \mid \leq j\right\}$. For a set $S$, the number of elements in $S$ is also denoted by $|S|$.

Definition 1 Let $\Sigma$ be a finite alphabet and $\Omega$ be a family of subsets of $\Sigma^{*}$ called a type over $\Sigma$. Let $V$ be a set whose elements are called motif components and $\tau$ be a mapping from $V$ to $\Omega$. For each motif component $x \in V$, the range of $x$ is the set $\tau(x)$ in $\Omega$ which specifies the set of strings in $\Sigma^{*}$ that the motif component $x$ can get. A motif $\pi$ of type $\Omega$ is an expression of the form

$$
\pi=\pi_{1} \cdots \pi_{n}
$$

where $\pi_{1}, \ldots, \pi_{n}$ are mutually distinct motif components. We denote by $L(\pi)$ the set of strings defined by $\left\{w_{1} \cdots w_{n} \mid w_{1} \in \tau\left(\pi_{1}\right), \ldots, w_{n} \in \tau\left(\pi_{n}\right)\right\}$. For a string $w$ and a motif $\pi$, we say that $\pi$ accepts (rejects) $w$ if $w \in L(\pi)(w \notin L(\pi))$.

A motif component $x$ whose range $\tau(x)$ consists of a single element, say $w \in \Sigma^{*}$, is called a constant and denoted by $w$ if no confusion occurs. On the other hand, if the range $\tau(x)$ consists of at least two elements, $x$ is called a variable. We often confuse a motif component with its range without any notice.

Example 1 A regular pattern [1] is an expression of the form $\pi=w_{0} x_{1} w_{1} \cdots w_{n-1} x_{n} w_{n}$ consisting of constants $w_{0}, \ldots, w_{n} \in \Sigma^{*}$ and distinct variables $x_{1}, \ldots, x_{n}$ with range $\Sigma^{+}$. The pattern $\pi$ defines a set $L(\pi)$ of strings in $\Sigma^{*}$ obtained by substituting any strings in $\Sigma^{+}$to the variables $x_{1}, \ldots, x_{n}$. Thus any regular pattern is regarded as a motif of type $\Omega=\left\{\Sigma^{+}\right\} \cup\left\{\{w\} \mid w \in \Sigma^{*}\right\}$.

Example 2 Let $\Sigma$ be the set of 20 symbols representing the amino acid residues. Let $\Omega_{1}=\{z \mid$ $\emptyset \neq z \subseteq \Sigma\}$ be the family of all nonempty subsets of $\Sigma$. Then the leucine zipper L- $x(6)-\mathrm{L}-x(6)-$ L- $x(6)-\mathrm{L}$, a 'helix-turn-helix' motif of some family of DNA binding proteins [LIVM]-x(1)-[DE][LIVM]-A- $x(2)-[S T A G]-x-V-[S P]-x(2)-[S T A G]-[L I V M A]-x(2)-[L I V M A]-[L I V M]$ and a motif such as [AC]$x(1)-\mathrm{V}-x(4)-\{$ ED $\}$ in PROSITE [3] can be regarded as motifs of type $\Omega_{1}$, where $x(i)$ represents 
any strings of length $i$ and the expressions like [LIVM] represents "L or I or V or M" and and $\{$ ED $\}$ represents "any but $\mathrm{E}$ or D." Note that $x(1)$ corresponds to $\Sigma \in \Omega_{1}$ and $x(i)$ is expressed as $\overbrace{x(1) \cdots x(1)}^{i}$. In order to express the zinc finger motif $\mathrm{C}-x(2,4)-\mathrm{C}-x(12)-\mathrm{H}-x(3,5)-\mathrm{H}$, we need $\Omega=\left\{\Sigma^{[i, j]} \mid 1 \leq i \leq j\right\} \cup \Omega_{1}$ as its type.

Since these motifs are embedded in sequences, we should add motif components with range $\Sigma^{+}$at the left and right ends of the motifs when we consider the whole sequences containing these motifs. Thus it suffices to consider the type $\Omega_{\infty}=\left\{\Sigma^{+}\right\} \cup\left\{\Sigma^{[i, j]} \mid 1 \leq i \leq j\right\} \cup\{z \mid \emptyset \neq$ $z \subseteq \Sigma\}$ for expressing motifs.

A yes-no example is a pair $(\alpha, \beta)$ of strings in $\Sigma^{*}$ with $\alpha \neq \beta$. For a motif $\pi$ and a yes-no example $(\alpha, \beta)$, we say that $(\alpha, \beta)$ is good for $\pi$ if $\pi$ accepts $\alpha$ but rejects $\beta$. A yes-no sample is a set $S=\left\{\left(\alpha^{(1)}, \beta^{(1)}\right), \ldots,\left(\alpha^{(m)}, \beta^{(m)}\right)\right\}$ of yes-no examples. We call strings $\alpha^{(1)}, \ldots, \alpha^{(m)}$ the positive examples of $S$ and strings $\beta^{(1)}, \ldots, \beta^{(m)}$ the negative examples of $S$. For a motif $\pi$ and a yes-no sample $S$, we define $\operatorname{cost}(S, \pi)$ to be the number of yes-no examples in $S$ which are good for $\pi$. Note that $\operatorname{cost}(S, \pi)=|L(\pi) \cap P| \times \mid\left(\Sigma^{*}-L(\pi) \cap N \mid\right.$ if a yes-no sample $S$ is provided as $P \times N$ with two disjoint sets $P$ and $N$ of strings.

Let $\Omega$ be a type over $\Sigma$. The best consensus motif problem for type $\Omega$ is, given a yes-no sample $S$, to find a motif $\pi$ of type $\Omega$ that maximizes $\operatorname{cost}(S, \pi)$.

\section{Best Consensus Motif Problem is Intractable}

This section investigates the complexity of finding a best consensus motif from a yes-no sample by considering two kinds of types. The one is

$$
\Omega_{1}=\{z \mid \emptyset \neq z \subseteq \Sigma\}
$$

which allows only simple motif components and the other is

$$
\Omega_{\infty}=\left\{\Sigma^{+}\right\} \cup\left\{\Sigma^{[i, j]} \mid 1 \leq i \leq j\right\} \cup\{z \mid \emptyset \neq z \subseteq \Sigma\}
$$

which involves a variety of expressions. We consider the decision version of the best consensus motif problem for $\Omega_{1}\left(\Omega_{\infty}\right)$, which is, given a yes-no sample $S$ and an integer $K \geq 0$, to decide if there is a motif $\pi$ of type $\Omega_{1}\left(\Omega_{\infty}\right)$ such that $\operatorname{cost}(S, \pi) \geq K$.

We start with an observation on the type $\Omega_{1}$. Let $S=\left\{\left(\alpha^{(1)}, \beta^{(1)}\right), \ldots,\left(\alpha^{(m)}, \beta^{(m)}\right)\right\}$ be a yes-no sample. We can assume that $\left|\alpha^{(1)}\right|=\cdots=\left|\alpha^{(m)}\right|$ since the length of a motif of type $\Omega_{1}$ must be the same as the length of $\alpha^{(i)}$ if $\left(\alpha^{(i)}, \beta^{(i)}\right)$ is good for $\mu$. The problem of finding, if any, a motif $\mu^{*}$ of type $\Omega_{1}$ such that all yes-no examples in $S$ are good for $\mu^{*}$ can be solved in polynomial time. This is because $\mu^{*}$ must be of the form $\mu^{*}=\tilde{\mu}_{1} \cdots \tilde{\mu}_{n}$ with $\tilde{\mu}_{k}=\left\{\alpha_{k}^{(i)} \mid 1 \leq i \leq m\right\}$ for $1 \leq k \leq n$ and $\mu^{*}$ must reject all negative examples from $S$, where $\alpha^{(i)}=\alpha_{1}^{(i)} \cdots \alpha_{n}^{(i)}$ with $\alpha_{1}^{(i)}, \ldots, \alpha_{n}^{(i)} \in \Sigma$. We do not know wheter the same observation holds for the type $\Omega_{\infty}$.

The main result in this section asserts that the best consensus motif problem is computationally intractable even if the type is simple $\left(\Omega_{1}\right)$ or ample $\left(\Omega_{\infty}\right)$.

\section{Theorem 1}

(1) The best consensus motif problem for $\Omega_{1}=\{z \mid \emptyset \neq z \subseteq \Sigma\}$ is NP-complete.

(2) The best consensus motif problem for $\Omega_{\infty}$ is NP-complete.

The results also hold even if $\Sigma=\{0,1\}$ and a yes-no sample $S$ is provided as $P \times N$ with two disjoint sets $P$ and $N$.

Proof. For a technical reason, we shall prove (2) first. The proof of (1) shall be given as a modified version of the proof of (2).

Obviously the problem is in NP. We shall show that there is a polynomial-time reduction from MAX2SAT [4], which is, given a formula in 2-CNF and a positive integer $K$, to decide if there is a truth assignment satisfying 


\begin{tabular}{|c|c|c|c|c|c|c|}
\hline & & $\begin{array}{l}(1) \\
2 n\end{array}$ & $\stackrel{(2)}{2 n}$ & & $\begin{array}{l}(M) \\
2 n\end{array}$ & \\
\hline$s_{1}$ & $=$ & $\overbrace{1000 \cdots 00}$ & $\overbrace{1000 \cdots 00}$ & $\cdots$ & $\overbrace{1000 \cdots 00}$ & \\
\hline$s_{2}$ & $=$ & $0100 \cdots 00$ & $0100 \cdots 00$ & $\cdots$ & $0100 \cdots 00$ & \\
\hline$\vdots$ & & $\cdot$ & $\cdot$ & $\vdots$ & $\cdot$ & \\
\hline$s_{2 n}$ & $=$ & $0000 \cdots 01$ & $0000 \cdots 01$ & $\cdots$ & $0000 \cdots 01$ & \\
\hline$e_{1}$ & $=$ & $0000 \cdots 00$ & $0000 \cdots 00$ & $\cdots$ & $0000 \cdots 00$ & \\
\hline$e_{2}$ & $=$ & $0000 \cdots 00$ & $0000 \cdots 00$ & $\cdots$ & $0000 \cdots 00$ & 0 \\
\hline$e_{3}$ & $=$ & $0000 \cdots 00$ & $0000 \cdots 00$ & $\cdots$ & $0000 \cdots 00$ & 00 \\
\hline$\vdots$ & & $\vdots$ & $\vdots$ & $\vdots$ & $\vdots$ & $\cdot$ \\
\hline & & $\overbrace{}^{2 n}$ & $\overbrace{}^{2 n}$ & & $\overbrace{}^{2 n}$ & ${ }^{L-1}$ \\
\hline$e_{L}$ & $=$ & $\overparen{0000 \cdots 00}$ & $\overparen{0000 \cdots 00}$ & $\cdots$ & $\overparen{0000 \cdots 00}$ & $\overparen{0000000 \cdots 0000}$ \\
\hline
\end{tabular}

Figure 1: Positive examples.

at least $K$ clauses. Let $F=C_{1} \cdots C_{m}$ be a formula in 2 -CNF with variables $u_{1}, u_{2}, \ldots, u_{n}$. We may assume without loss of generality that any clause does not contain both $u_{k}$ and $\overline{u_{k}}$ for any $1 \leq k \leq n$. We shall define $P$ of positive examples and $N$ of negative examples such that at least $K$ clauses of $F$ are satisfiable if and only if there is a motif $\pi$ with $\operatorname{cost}(S, \pi) \geq(n+L)(3 n M+K)$, where $S=P \times N, L=n^{2}-n+2$ and $M=(2 n+L) m+1$. Let $K^{\prime}=(n+L)(3 n M+K)$. The set $P$ consists of the following $2 n+L$ positive examples $s_{1}, \ldots, s_{2 n}, e_{1}, \ldots, e_{L}$ and the set $N$ consists of the following $3 n M+m$ negative examples $t_{1}^{1}, \ldots, t_{M}^{n}, h_{1}^{1}, \ldots, h_{M}^{2 n}, d_{1}, \ldots, d_{m}$ (see Fig. 1 and Fig. 2):

$$
\begin{aligned}
& s_{i}=\left(0^{i-1} 10^{2 n-i}\right)^{M} \quad \text { for } 1 \leq i \leq 2 n \text {, } \\
& e_{i}=0^{2 n M+i-1} \quad \text { for } 1 \leq i \leq L, \\
& t_{j}^{k}=\left(0^{2 n}\right)^{i-1} \cdot(00)^{k-1} 11(00)^{n-k} \cdot\left(0^{2 n}\right)^{M-i} \\
& \begin{array}{r}
\text { for } 1 \leq k \leq n, 1 \leq j \leq M, \\
h_{j}^{i}=\left(0^{2 n}\right)^{j-1} \cdot\left(0^{i-1} \cdot 1 \cdot 0^{2 n-i}\right) \cdot\left(0^{2 n}\right)^{M-j-1} \cdot 0^{2 n-1} \\
\text { for } 1 \leq i \leq 2 n, 1 \leq j<M,
\end{array} \\
& h_{M}^{i}=\left(0^{2 n}\right)^{M-1} \cdot 0^{i-1} \cdot 1 \cdot 0^{2 n-i-1} \quad \text { for } 1 \leq i<2 n \text {, } \\
& h_{M}^{2 n}=0^{2 n M-1} \text {, } \\
& d_{i}=\left(r_{1}^{i} r_{2}^{i} \ldots r_{n}^{i}\right)^{M} \quad \text { for } 1 \leq i \leq m \text {, where } \\
& r_{k}^{i}= \begin{cases}10 & \text { if literal } u_{k} \text { appears in } C_{i} \\
01 & \text { if literal } \overline{u_{k}} \text { appears in } C_{i} \\
0 & \text { otherwise. }\end{cases}
\end{aligned}
$$

Note that positive and negative examples are strings over $\Sigma=\{0,1\}$. Therefore $\Omega_{\infty}$ consists of $\{0\},\{1\}$, $\{0,1\}^{+}$and $\{0,1\}^{[i, j]}$ for $j \geq i \geq 1$. In the following argument, $\omega^{+}$represents a variable whose range is $\{0,1\}^{+}$ and $\omega^{[i, j]}$ represents a variable whose range is $\{0,1\}^{[i, j]}$ for $j \geq i \geq 1$. The constants are 0 and 1 . Let $X$ denote the set of variables. For convenience, we denote by $X \cup 0$ the set consisting of all variables and constants 0 .

Suppose that at least $K$ clauses of $F$ are satisfied by a truth assignment $\hat{u}_{1}, \ldots, \hat{u}_{n}$. Then we define a motif $\pi=\left(\tau_{1} \ldots \tau_{n}\right)^{M}$ by putting $\tau_{k}=0 \omega^{+}$if $\hat{u}_{k}=$ true and $\tau_{k}=\omega^{+} 0$ if $\hat{u}_{k}=$ false for $1 \leq k \leq n$. Obviously, either $s_{2 k-1}$ or $s_{2 k}$ is accepted by $\pi$ for $1 \leq k \leq n$, and all $e_{1}, \ldots, e_{L}$ are accepted by $\pi$. On the other hand, $\pi$ rejects $t_{j}^{k}$ for all $1 \leq k \leq n, 1 \leq j \leq M$ and all $h_{j}^{i}$ for all $1 \leq i \leq 2 n, 1 \leq j \leq M$. By the definition of $r_{k}^{i}$, a clause $C_{i}$ of $F$ is satisfied by the truth assignment $\hat{u}_{1}, \ldots, \hat{u}_{n}$ if and only if $\bar{d}_{j}$ is rejected by $\pi$. Thus the number of clauses satisfied by the truth assignment is equal to the number of $d_{j}$ 's rejected by $\pi$. Then $\operatorname{cost}(S, \pi) \geq K^{\prime}$.

Conversely, we suppose that there exists a motif $\pi$ with $\operatorname{cost}(S, \pi) \geq K^{\prime}$.

First, we show Claim 1 below. For Claim 1, we introduce the following conventions. A motif is denoted as $\pi=\theta_{0} y_{1} \theta_{1} \cdots \theta_{n-1} y_{n} \theta_{n}$, where $y_{i}$ is a variable in $X$ for $1 \leq i \leq n$ and $\theta_{j}$ is a finite (possibly empty) sequence of constants 0 and 1 for $0 \leq j \leq n$. For a variable $y_{k} \in X$, let $\left\|y_{k}\right\|=\min \left\{|w| \mid w \in y_{k}\right\}$ for $1 \leq k \leq n$. Then we denote by $\|\pi\|=\left|\theta_{0}\right|+\left\|y_{1}\right\|+\left|\theta_{1}\right|+\cdots\left|\theta_{n-1}\right|+\left\|y_{n}\right\|+\left|\theta_{n}\right|$. For $\pi=\theta_{0} y_{1} \theta_{1} \cdots \theta_{n-1} y_{n} \theta_{n}$, a tuple $\varphi=\left[y_{1} \leftarrow w_{1}, \ldots, y_{n} \leftarrow w_{n}\right]$ of assignments of strings $w_{i} \in y_{i}$ to the variable occurrences $y_{i}$ for $1 \leq i \leq n$ is called a fill-in of $\pi$. Let $w=\theta_{0} w_{1} \theta_{1} \cdots \theta_{n-1} w_{n} \theta_{n}$ be the string, denoted by $\pi \varphi$, obtained by the fill-in $\varphi$. We say that an occurrence of a substring $w^{\prime}$ in $w$ is covered by the variable occurrence $y_{i}$ under $\varphi$ if $w^{\prime}$ is contained in $w_{i}$.

ClaIm 1. If a motif $\pi$ satisfies $\operatorname{cost}(S, \pi) \geq K^{\prime}$, then $\pi \in(X \cup 0)^{+}$and $\|\pi\|=2 n M$. 


\begin{tabular}{|c|c|c|c|c|c|c|}
\hline & & $\overbrace{2 n}^{(1)}$ & $2_{2 n}^{(2)}$ & & & $\overbrace{}^{(M)}{ }^{(M)}$ \\
\hline$t_{1}^{1}$ & $=$ & $\overbrace{1100 \cdots 00}$ & $\overbrace{0000 \cdots 00}$ & $\cdots$ & $\cdots$ & $\overbrace{0000 \cdots 00}$ \\
\hline$t_{2}^{1}$ & $=$ & $0000 \cdots 00$ & $1100 \cdots 00$ & $\cdots$ & $\cdots$ & $0000 \cdots 00$ \\
\hline$\vdots$ & & $\vdots$ & $\vdots$ & $\vdots$ & & $\vdots$ \\
\hline$t_{M}^{1}$ & $=$ & $0000 \cdots 00$ & $0000 \cdots 00$ & $\cdots$ & $\cdots$ & $1100 \cdots 00$ \\
\hline$t_{1}^{2}$ & $=$ & $0011 \cdots 00$ & $0000 \cdots 00$ & $\cdots$ & $\ldots$ & $0000 \cdots 00$ \\
\hline$t_{2}^{2}$ & $=$ & $0000 \cdots 00$ & $0011 \cdots 00$ & $\cdots$ & $\cdots$ & $0000 \cdots 00$ \\
\hline$\vdots$ & & $\vdots$ & $\vdots$ & $\vdots$ & & $\vdots$ \\
\hline$t_{M}^{2}$ & $=$ & $\begin{array}{c}00 \cdots 0011 \\
\vdots\end{array}$ & $0000 \cdots 00$ & $\cdots$ & $\cdots$ & $0011 \cdots 00$ \\
\hline$t_{1}^{n}$ & $=$ & $00 \cdots 0011$ & $00 \cdots 0000$ & $\cdots$ & $\cdots$ & $00 \cdots 0000$ \\
\hline$t_{2}^{n}$ & $=$ & $00 \cdots 0000$ & $00 \cdots 0011$ & $\cdots$ & $\cdots$ & $00 \cdots 0000$ \\
\hline$\vdots$ & & $\vdots$ & $\vdots$ & $\vdots$ & & $\vdots$ \\
\hline$t_{M}^{n}$ & $=$ & $00 \cdots 0000$ & $00 \cdots 0000$ & $\cdots$ & $\cdots$ & $00 \cdots 0011$ \\
\hline$d_{1}$ & $=$ & $r_{1}^{1} r_{2}^{1} \cdots r_{n}^{1}$ & $r_{1}^{1} r_{2}^{1} \cdots r_{n}^{1}$ & $\cdots$ & & $r_{1}^{1} r_{2}^{1} \cdots r_{n}^{1}$ \\
\hline$d_{2}$ & $=$ & $r_{1}^{2} r_{2}^{2} \cdots r_{n}^{2}$ & $r_{1}^{2} r_{2}^{2} \cdots r_{n}^{2}$ & $\cdots$ & & $r_{1}^{2} r_{2}^{2} \cdots r_{n}^{2}$ \\
\hline$\vdots$ & & $\vdots$ & $\vdots$ & $\vdots$ & & $\vdots$ \\
\hline$d_{m}$ & $=$ & $r_{1}^{m} r_{2}^{m} \cdots r_{n}^{m}$ & $r_{1}^{m} r_{2}^{m} \cdots r_{n}^{m}$ & $\cdots$ & & $r_{1}^{m} r_{2}^{m} \cdots r_{n}^{m}$ \\
\hline & & $\overbrace{}^{2 n}$ & $\overbrace{}^{2 n}$ & & $\overbrace{\overbrace{}^{2 n}}^{2 n}$ & $\overbrace{}^{2 n-1}$ \\
\hline$h_{1}^{1}$ & $=$ & $\overbrace{100 \cdots 000}$ & $\overbrace{000 \cdots 000}$ & $\cdots$ & $\overbrace{000 \cdots 000}$ & $\overbrace{000 \cdots 00}$ \\
\hline$h_{2}^{1}$ & $=$ & $000 \cdots 000$ & $100 \cdots 000$ & $\cdots$ & $000 \cdots 000$ & $000 \cdots 00$ \\
\hline$\vdots$ & & $\vdots$ & $\vdots$ & $\vdots$ & & $\vdots$ \\
\hline$h_{M}^{1}$ & $=$ & $000 \cdots 000$ & $000 \cdots 000$ & $\cdots$ & $000 \cdots 000$ & $100 \cdots 00$ \\
\hline & & $:$ & & & & \\
\hline $\begin{array}{l}h_{1}^{2 n-1} \\
h^{2 n-1}\end{array}$ & $=$ & $000 \cdots 010$ & $000 \cdots 000$ & $\cdots$ & $000 \cdots 000$ & $000 \cdots 00$ \\
\hline$h_{2}^{2 n-1}$ & $=$ & $000 \cdots 000$ & $000 \cdots 010$ & $\cdots$ & $000 \cdots 000$ & $000 \cdots 00$ \\
\hline$\vdots$ & & $\vdots$ & $\vdots$ & $\vdots$ & & $\vdots$ \\
\hline$h_{M-1}^{2 n-1}$ & $=$ & $000 \cdots 000$ & $000 \cdots 000$ & $\cdots$ & $000 \cdots 010$ & $000 \cdots 00$ \\
\hline$h_{M}^{2 n-1}$ & $=$ & $000 \cdots 000$ & $000 \cdots 000$ & $\cdots$ & $000 \cdots 000$ & $000 \cdots 01$ \\
\hline$h_{1}^{2 n}$ & $=$ & $000 \cdots 001$ & $000 \cdots 000$ & $\ldots$ & $000 \cdots 000$ & $000 \cdots 00$ \\
\hline$h_{2}^{2 n}$ & $=$ & $000 \cdots 000$ & $000 \cdots 001$ & $\cdots$ & $000 \cdots 000$ & $000 \cdots 00$ \\
\hline$\vdots$ & & $\vdots$ & $\vdots$ & $\vdots$ & & $\vdots$ \\
\hline$h_{M-1}^{2 n}$ & $=$ & $000 \cdots 000$ & $000 \cdots 000$ & $\cdots$ & $000 \cdots 001$ & $000 \cdots 00$ \\
\hline$h_{M}^{2 n}$ & $=$ & $000 \cdots 000$ & $000 \cdots 000$ & $\ldots$ & $000 \cdots 000$ & $000 \cdots 00$ \\
\hline
\end{tabular}

Figure 2: Negative examples. 
Proof. If 1 occurs in $\pi$, then $\pi$ rejects all $e_{i}$ for $1 \leq i \leq L$. Then $\operatorname{cost}(S, \pi) \leq 2 n(3 n M+m)<K^{\prime}$, by the definition of $M$ and $K^{\prime}$. Thus $\pi \in(X \cup 0)^{+}$. If $\pi$ rejects all $s_{i}$ for $1 \leq i \leq 2 n$, then $\operatorname{cost}(S, \pi) \leq L(3 n M+m)<K^{\prime}$. Therefore, $\|\pi\| \leq 2 n M$ and $s_{i}$ is in $L(\pi)$ for some $1 \leq i \leq 2 n$.

Suppose now that $\|\pi\| \leq 2 n M-1$. Let $\pi=\theta_{0} y_{1} \theta_{1} \cdots y_{p} \theta_{p}$. Since $s_{i}$ is accepted by $\pi$, there is a fill-in $\varphi=\left[y_{1} \leftarrow \xi_{1}, \ldots, y_{p} \leftarrow \xi_{p}\right]$ such that $\pi \varphi=s_{i}$.

Fact 1. If $\pi$ accepts $s_{i}$, then $\pi$ accepts $h_{j}^{i}$ or $h_{j}^{i-1}$ for each $1 \leq j \leq M$, where $h_{j}^{0}=h_{j-1}^{2 n}$.

Proof. Note that $s_{i}$ contains exactly $M$ occurrences of 1 . For each $1 \leq j \leq M$, we consider the $j$ th occurrence of 1 in $s_{i}$. Let $y_{q}$ be the variable occurrence covering the $j$ th occurrence of 1 in $s_{i}$ under $\varphi$. There are two cases according to the location of the $j$ th occurrence of 1 in $\xi_{q}$.

(1) $\xi_{q}=1$ : Since $\|\pi\| \leq 2 n M-1$ and $\left|s_{i}\right|=2 n M$, there is some $r$ with $\left|\xi_{r}\right| \geq 2$ such that $y_{r}=\omega^{+}$or $y_{r}=\omega^{\left[l_{1}, l_{2}\right]}$ with $l_{1}<\left|\xi_{r}\right| \leq l_{2}$. When $q<r$, let $\xi_{q}^{\prime}=1, \xi_{r}^{\prime}=0^{\left|\xi_{r}\right|-1}$, and $\xi_{l}^{\prime}=0^{\left|\xi_{l}\right|}(l \neq q, r)$. Then for $\varphi^{\prime}=\left[y_{1} \leftarrow \xi_{1}^{\prime}, \ldots, y_{p} \leftarrow \xi_{p}^{\prime}\right]$ we have $\pi \varphi^{\prime}=h_{j}^{i}$. When $r<q$, let $\xi_{q}^{\prime}=1, \xi_{r}^{\prime}=0^{\left|\xi_{r}\right|-1}$, and $\xi_{l}^{\prime}=0^{\left|\xi_{l}\right|}(l \neq q, r)$. Then for $\varphi^{\prime}=\left[y_{1} \leftarrow \xi_{1}^{\prime}, \ldots, y_{p} \leftarrow \xi_{p}^{\prime}\right]$ we have $\pi \varphi^{\prime}=h_{j}^{i-1}$.

(2) $\xi_{q}=\gamma 10 \gamma^{\prime}$ or $\xi_{q}=\gamma 01 \gamma^{\prime}$ : If $y_{q}=\omega^{+}$or $y_{q}=\omega^{\left[l_{1}, l_{2}\right]}$ with $l_{1}<\left|\xi_{q}\right|$, then let $\xi_{q}^{\prime}=0^{|\gamma|} 10^{\left|\gamma^{\prime}\right|}$ and $\xi_{l}^{\prime}=0^{\left|\xi_{l}\right|}$ $(l \neq q)$ and let $\varphi^{\prime}=\left[y_{1} \leftarrow \xi_{1}^{\prime}, \ldots, y_{p} \leftarrow \xi_{p}^{\prime}\right]$. Then if $\xi_{q}=\gamma 10 \gamma^{\prime}$, then $\pi \varphi^{\prime}=h_{j}^{i}$. If $\xi_{q}=\gamma 01 \gamma^{\prime}$, then $\pi \varphi^{\prime}=h_{j}^{i-1}$. If $y_{q}=\omega^{\left[l_{1}, l_{2}\right]}$ with $l_{1}=\left|\xi_{q}\right|$, then by the same argument as (1) we can conclude that $\pi$ accepts $h_{j}^{i}$ or $h_{j}^{i-1}$. $\square$

Fact 2. If $\pi$ accepts $b$ positive examples from $s_{1}, \ldots, s_{2 n}$, then $\pi$ also accepts at least $\left\lfloor\frac{b}{2}\right\rfloor \cdot M$ negative examples from $h_{1}^{1}, \ldots, h_{M-1}^{2 n}$.

Proof. Let $s_{i_{1}}, \ldots, s_{i_{b}}$ be positive examples accepted by $\pi$, where $i_{1}<i_{2}<\cdots<i_{b}$. By Fact 1 , for each $s_{i_{k}}, \pi$ accepts $M$ distinct negative examples $h_{j}^{i_{k}-1}$ or $h_{j}^{i_{k}}$ for $1 \leq j \leq M$. Let $H_{k}$ be the set of these $M$ negative examples $h_{j}^{i_{k}-1}$ or $h_{j}^{i_{k}}(1 \leq j \leq M)$ accepted by $\pi$ for $s_{i_{k}}$.

Then $H_{1}, H_{3}, \ldots, H_{2 \cdot\left\lfloor\frac{b}{2}\right\rfloor+1}$ are pairwise disjoint. Therefore $\left|H_{1} \cup H_{3} \cup \cdots \cup H_{2 \cdot\left\lfloor\frac{b}{2}\right\rfloor+1}\right|=\left\lfloor\frac{b}{2}\right\rfloor \cdot M$.

By Fact 2 , if $\pi$ accepts $b$ positive examples from $s_{1}, \ldots, s_{2 n}$, then

$$
\operatorname{cost}(S, \pi) \leq(L+b)\left(\left(3 n-\left\lfloor\frac{b}{2}\right\rfloor\right) M+m\right)
$$

since at least $\left\lfloor\frac{b}{2}\right\rfloor \cdot M$ negative examples from $h_{1}^{1}, \ldots, h_{M-1}^{2 n}$ are accepted by $\pi$. By easy but tedious calculations, we can show that $(L+b)\left(\left(3 n-\left\lfloor\frac{b}{2}\right\rfloor\right) M+m\right)<K^{\prime}$ for all $1 \leq b \leq 2 n$. This is again a contradiction. Thus $\|\pi\|$ must be exactly $2 n M$.

Claim 2. If $\operatorname{cost}(S, \pi) \geq K^{\prime}, \pi$ accepts at least $n$ positive examples from $s_{1}, \ldots, s_{2 n}$.

Proof. Otherwise, $\operatorname{cost}(S, \pi) \leq(L+n-1)(3 n M+m)<K^{\prime}$.

ClaIM 3. Let $\pi$ be a motif that maximizes $\operatorname{cost}(S, \pi)$. If $\operatorname{cost}(S, \pi) \geq K^{\prime}$, then $\pi$ can be described as a motif of type $\left\{\{0,1\}^{+}\right\}$having the form

$$
\pi=\left(\pi_{1} \pi_{2} \cdots \pi_{2 n}\right)^{M}
$$

where $\pi_{2 k-1} \pi_{2 k}=0 \omega^{+}$or $\pi_{2 k-1} \pi_{2 k}=\omega^{+} 0$ for $1 \leq k \leq n$.

Proof. By Claim 1, the motif $\pi$ satisfies $\|\pi\|=2 n M$ but may contain variables and $\omega^{\left[l_{1}, l_{2}\right]}$. However, by $|\tilde{\pi}|=2 n M$ and $\tilde{\pi}$ accepts the same positive examples in $P$ as $\pi$ and $\tilde{\pi}$ rejects the same negative examples in $N$ as $\pi$. Let $\pi$ be this $\tilde{\pi}$. Since $|\pi|=2 n M, \pi$ is written as $\pi=\pi_{1} \pi_{2} \cdots \pi_{2 n M}$ with $\pi_{i} \in X$ or $\pi_{i}=0$ for $1 \leq i \leq 2 n M$. For each $1 \leq k \leq n$, we consider positive examples $s_{2 k-1}$ and $s_{2 k}$ and segments $\pi_{2 n(i-1)+2 k-1} \pi_{2 n(i-1)+2 k}$ for $1 \leq i \leq M$.

Case 1. $s_{2 k-1} \in L(\pi)$ and $s_{2 k} \in L(\pi)$ : Then $\pi_{2 n(i-1)+2 k-1} \pi_{2 n(i-1)+2 k}=\omega^{+} \omega^{+}$for all $1 \leq i \leq M$. If we replace $\pi_{2 n(i-1)+2 k-1} \pi_{2 n(i-1)+2 k}$ with $0 \omega^{+}$(or $\left.\omega^{+} 0\right)$ in $\pi$ for all $1 \leq i \leq M$. Let $\pi^{\prime}$ be the resulting motif obtained from $\pi$ by this replacement. Then $\pi^{\prime}$ rejects $s_{2 k-1}$ (or $s_{2 k}$ ) and $M$ negative examples $t_{1}^{k}, \ldots, t_{M}^{k}$ in addition to all positive and negative examples rejected by $\pi$. On the other hand, $\pi$ accepts all $e_{i}$ for $1 \leq i \leq L$ since $\pi \in(X \cup 0)^{+}$. Moreover, by Claim 2, $\pi$ must accepts at least $n$ positive examples from $s_{1}, \ldots, s_{2 n}$. By assumption, $\pi$ accepts $s_{2 k-1}, s_{2 k}$ and $t_{(k-1) M+1}, \ldots, t_{k M}$. Therefore $\operatorname{cost}\left(S, \pi^{\prime}\right)-\operatorname{cost}(S, \pi) \geq(n+L) M-1 \cdot((3 n-1) M+m)>0$, by the definition of $L$ and $M$. This contradicts that $\pi$ maximizes $\operatorname{cost}(S, \pi)$.

Case 2. $s_{2 k-1} \notin L(\pi)$ and $s_{2 k} \notin L(\pi)$ : By Claim 2, $\pi$ must accept at least $n$ positive examples from $s_{1}, \ldots, s_{2 n}$. Therefore there is some $1 \leq j \leq n$ such that $s_{2 j-1} \in L(\pi)$ and $s_{2 j} \in L(\pi)$. But this does not hold from Case 1.

Thus either $s_{2 k-1} \in L(\pi)$ or $s_{2 k} \in L(\pi)$ for $1 \leq k \leq n$.

Case 3. $s_{2 k-1} \in L(\pi)$ and $s_{2 k} \notin L(\pi)$ : Then $\pi_{2 n(i-1)+2 k-1} \pi_{2 n(i-1)+2 k}$ is either $\omega^{+} \omega^{+}$or $\omega^{+} 0$ for $1 \leq$ $i \leq M$. If $\pi_{2 n(i-1)+2 k-1} \pi_{2 n(i-1)+2 k}=\omega^{+} \omega^{+}$for some $1 \leq i \leq M$, then we replace $\pi_{2 n(i-1)+2 k-1} \pi_{2 n(i-1)+2 k}$ with $\omega^{+} 0$ in $\pi$. Let $\pi^{\prime}$ be the resulting motif. Then $\pi^{\prime}$ does not reject any positive examples which are 
accepted by $\pi$ and $\pi^{\prime}$ does not accept any negative examples which are rejected by $\pi$. In addition, $\pi^{\prime}$ rejects $t_{i}^{k}$. Therefore $\operatorname{cost}\left(S, \pi^{\prime}\right)>\operatorname{cost}(S, \pi)$. This also contradicts the hypothesis that $\operatorname{cost}(S, \pi)$ is maximum. Thus $\pi_{2 n(i-1)+2 k-1} \pi_{2 n(i-1)+2 k}=\omega^{+} 0$ for all $1 \leq i \leq M$.

Case 4. $s_{2 k-1} \notin L(\pi)$ and $s_{2 k} \in L(\pi)$ : In the same way as Case 3 , we can see that $\pi_{2 n(i-1)+2 k-1} \pi_{2 n(i-1)+2 k}=$ $0 \omega^{+}$for all $1 \leq i \leq M$.

By Cases 1-4, we see that $\pi$ is written as $\left(\pi_{1} \pi_{2} \cdots \pi_{2 n}\right)^{M}$ with $\pi_{2 k-1} \pi_{2 k}=0 \omega^{+}$or $\pi_{2 k-1} \pi_{2 k}=\omega^{+} 0$ for $1 \leq k \leq n$.

By Claim 3, there is a motif $\pi=\left(\pi_{1} \cdots \pi_{2 n}\right)^{M}$ such that $\operatorname{cost}(S, \pi) \geq K^{\prime}$ and $\pi_{2 k-1} \pi_{2 k}$ is $0 \omega^{+}$or $\omega^{+} 0$ for $1 \leq k \leq n$. The motif $\pi$ accepts either $s_{2 k-1}$ or $s_{2 k}$ for each $1 \leq k \leq n$ and accepts all other positive examples while $\pi$ rejects at least all negative examples except $d_{1}, \ldots, d_{m}$. Since $\operatorname{cost}(S, \pi) \geq K^{\prime}=(n+L)(3 n M+K)$, at least $K$ negative examples from $d_{1}, \ldots, d_{m}$ must be rejected by $\pi$. Then we define a truth assignment $\hat{u}_{k}=t r u e$ if $\pi_{2 k-1} \pi_{2 k}=0 \omega^{+}$and $\hat{u}_{k}=$ false if $\pi_{2 k-1} \pi_{2 k}=\omega^{+} 0$ for $1 \leq k \leq n$. Clearly, at least $K$ clauses $C_{i}$ are satisfied by this truth assignment. This completes the proof of (2).

We now give a proof of (1). Since the basic idea of the proof is similar to that of (2), we shall give only the sketch. Since the type is $\Omega_{1}$, any variable occurrence in a motif gets a symbol in $\Sigma$. For a 2-CNF formula $F=C_{1} \cdots C_{m}$ with $n$ variables and an integer $K$, let $M^{\prime}=(2 n-1) m+1$. Then let $P^{\prime}$ be the set of positive examples consisting of $e_{1}^{\prime}, s_{1}^{\prime}, \ldots, s_{2 n}^{\prime}$, where $e_{1}^{\prime}, s_{1}^{\prime}, \ldots, s_{2 n}^{\prime}$ are the strings in $\{0,1\}^{*}$ obtained from $e_{1}, s_{1}, \ldots, s_{2 n}$ in Fig. 1 by replacing $M$ with $M^{\prime}$. Similarly, let $N^{\prime}$ be the set of negative examples consisting of $t^{\prime}{ }_{1}^{1}, \ldots, t^{\prime n}{ }^{\prime}$, and $d_{1}^{\prime}, \ldots, d_{m}^{\prime}$ obtained from $t_{1}^{1}, \ldots, t_{M}^{n}$ and $d_{1}, \ldots, d_{m}$ in Fig. 2 by replacing $M$ with $M^{\prime}$. Let $K^{\prime \prime}=(n+1)\left(n M^{\prime}+K\right)$. Assume that there is a motif $\pi$ of type $\Omega_{1}$ such that $\operatorname{cost}\left(S^{\prime}, \pi\right) \geq K^{\prime \prime}$. Let $\pi$ be a motif that maximizing $\operatorname{cost}\left(S^{\prime}, \pi\right)$. If $\pi$ contains constant 1 , then at most one positive example is accepted by $\pi$. In this case, $\operatorname{cost}\left(S^{\prime}, \pi\right) \leq n M^{\prime}+m<K^{\prime \prime}$, a contradiction. Then by an argument similar to Claim 3 , we can show that $\pi$ must be of the form $\pi=\left(\eta_{1} \cdots \eta_{n}\right)^{M^{\prime}}$ with $\eta_{j}=0 \omega^{+}$or $\eta_{j}=\omega^{+} 0$ for $1 \leq j \leq n$. The rest of the proof is similar and left to the reader. This completes the proof of (1).

\section{Approximating the Best Consensus Motif Problem for $\Omega_{1}$}

From Theorem 1, the best consensus motif problem for $\Omega_{1}=\{z \mid \emptyset \neq z \subseteq \Sigma\}$ is hardly solvable in polynomial time. This section presents a polynomial time greedy algorithm for this problem and its performance analysis by exploiting the probabilistic method due to Yannakakis [14] which was first applied to the maximum satisfiability problem. Since we consider only sets in $\Omega_{1}$, we assume that a yes-no sample $S=\left\{\left(\alpha^{(1)}, \beta^{(1)}\right), \ldots,\left(\alpha^{(m)}, \beta^{(m)}\right)\right\}$ satisfies $\left|\alpha^{(1)}\right|=\left|\beta^{(1)}\right|=$ $\cdots=\left|\alpha^{(m)}\right|=\left|\beta^{(m)}\right|$. For convenience, we identify the range of a motif component with the motif component itself.

For $1 \leq k \leq n$, we assume probabilities $p_{k}(z)$ for $z \in \Omega_{1}$, i.e., $0 \leq p_{k}(z) \leq 1$ and $\sum_{z \in \Omega_{1}} p_{k}(z)=1$. Let $\pi_{k}$ be a random variable taking a value $z$ in $\Omega_{1}$ with probability $p_{k}(z)$. We call an expression $\rho_{k}=z_{1} \cdots z_{k-1} \pi_{k} \cdots \pi_{n}$ a random motif of length $n$ with fixed motif components $z_{1}, \ldots, z_{k-1} \in \Omega_{1}$ and random variables $\pi_{k}, \ldots, \pi_{n}$. For a random motif $z_{1} \cdots z_{k-1} \pi_{k} \cdots \pi_{n}$, we denote by $P\left((\alpha, \beta), z_{1} \cdots z_{k-1} \pi_{k} \cdots \pi_{n}\right)$ the probability that a yes-no example $(\alpha, \beta)$ is good for $z_{1} \cdots z_{k-1} \pi_{k} \cdots \pi_{n}$. Formally, let

$$
H\left((\alpha, \beta), z_{1} \cdots z_{k-1} \pi_{k} \cdots \pi_{n}\right)=\left\{\left(y_{k}, \ldots, y_{n}\right) \in \Omega_{1}^{n-k+1} \mid(\alpha, \beta) \text { is good for } z_{1} \cdots z_{k-1} y_{k} \cdots y_{n}\right\} \text {. }
$$

Then $P\left((\alpha, \beta), z_{1} \cdots z_{k-1} \pi_{k} \cdots \pi_{n}\right)$ is given by

$$
\sum_{\left(y_{1}, \ldots, y_{n}\right) \in H\left((\alpha, \beta), z_{1} \cdots z_{k-1} \pi_{k} \cdots \pi_{n}\right)} p_{k}\left(y_{k}\right) \cdots p_{n}\left(y_{n}\right) .
$$

It requires exponential time with respect to $n$ if we simply calculate the above formula. However, it is polynomial time computable as shown below.

For $\sigma \in \Sigma$, let $S_{\sigma}=\left\{z \in \Omega_{1} \mid \sigma \in z\right\}$ and $p_{k}\left(S_{\sigma}\right)=\sum_{z \in S_{\sigma}} p_{k}(z)$. For a string $\gamma=\gamma_{1} \cdots \gamma_{n}$, let $\delta\left(\gamma, z_{1} \cdots z_{k-1}\right)=1$ if $\gamma_{1} \cdots \gamma_{k-1} \in z_{1} \cdots z_{k-1}$ else 0 . Then for a yes-no example $(\alpha, \beta)$ $\left(\alpha=\alpha_{1} \cdots \alpha_{n}, \beta=\beta_{1} \cdots \beta_{n}\right)$, the probability that $(\alpha, \beta)$ is good for $z_{1} \cdots z_{k-1} \pi_{k} \cdots \pi_{n}$ is 
Input: a yes-no sample $S$ and propabilities $p_{1}(z), \ldots, p_{n}(z)$ for $z \in \Omega_{1}$.

Output: a motif $\hat{\pi}=z_{1} \cdots z_{n}$.

$/ * \pi_{1}, \ldots, \pi_{n}$ are random variables with $p_{1}, \ldots, p_{n}$, respectively. $* /$

for $k \leftarrow 1$ to $n$

begin

Find $z \in \Omega_{1}$ maximizing $E\left(S, z_{1} \cdots z_{k-1} z \pi_{k+1} \cdots \pi_{n}\right)$;

$z_{k} \leftarrow z$

end

Figure 3: Greedy algorithm.

expressed as:

$$
\begin{aligned}
& P\left((\alpha, \beta), z_{1} \cdots z_{k-1} \pi_{k} \cdots \pi_{n}\right) \\
& \quad=\delta\left(\alpha, z_{1} \cdots z_{k-1}\right) \cdot \prod_{j \in\left\{j \geq k \mid \alpha_{j}=\beta_{j}\right\}} p_{j}\left(S_{\alpha_{j}}\right) \cdot\left(\prod_{j \in\left\{j \geq k \mid \alpha_{j} \neq \beta_{j}\right\}} p_{j}\left(S_{\alpha_{j}}\right)-\delta\left(\beta, z_{1} \cdots z_{k-1}\right) \cdot \prod_{j \in\left\{j \geq k \mid \alpha_{j} \neq \beta_{j}\right\}} p_{j}\left(S_{\alpha_{j}} \cap S_{\beta_{j}}\right)\right) \\
& \quad=\delta\left(\alpha, z_{1} \cdots z_{k-1}\right) \cdot\left(\prod_{j=k}^{n} p_{j}\left(S_{\alpha_{j}}\right)-\delta\left(\beta, z_{1} \cdots z_{k-1}\right) \cdot \prod_{j=k}^{n} p_{j}\left(S_{\alpha_{j}} \cap S_{\beta_{j}}\right)\right) .
\end{aligned}
$$

This can be computed in polynomial time with respect to $n$. Therefore, for a yes-no sample $S$, the expectation

$$
E\left(S, z_{1} \cdots z_{k-1} \pi_{k} \cdots \pi_{n}\right)=\sum_{(\alpha, \beta) \in S} P\left((\alpha, \beta), z_{1} \cdots z_{k-1} \pi_{k} \cdots \pi_{n}\right)
$$

is also computable in polynomial time with respect to $n$ and $m=|S|$.

Our algorithm for the best consensus motif problem for $\Omega_{1}$ is very simple as shown in Fig. 3 . We shall give a rigorous analysis of the algorithm and prove a lower bound of its performance.

Theorem 2 Let $s=|\Sigma|$. If the probability distributions $p_{1}, \ldots, p_{n}$ on $\Omega_{1}$ satisfy the following conditions for $1 \leq k \leq n$,

1. $p_{k}\left(S_{\sigma}\right) \leq \frac{s+1}{2 s}$ for all $\sigma$ in $\Sigma$,

2. $p_{k}\left(S_{\sigma} \cap S_{\tau}\right) \geq \frac{s+2}{4 s}$ for all $\sigma, \tau$ in $\Sigma$,

then

$$
\operatorname{cost}(S, \hat{\pi}) \geq E\left(S, \pi_{1} \cdots \pi_{n}\right)
$$

where $\hat{\pi}$ is a motif of type $\Omega_{1}$ produced by the algorithm in Fig. 3 for a yes-no sample $S$ and $\pi_{k}$ is a random variable taking a value $z$ with probablity $p_{k}(z)$ for $z \in \Omega_{1}(1 \leq k \leq n)$.

Proof. Let $S=\left\{\left(\alpha^{(1)}, \beta^{(1)}\right), \ldots,\left(\alpha^{(m)}, \beta^{(m)}\right)\right\}$ be a yes-no sample. We denote $\alpha^{(i)}$ and $\beta^{(i)}$ as $\alpha^{(i)}=\alpha_{1}^{(i)} \cdots \alpha_{n}^{(i)}$ and $\beta^{(i)}=\beta_{1}^{(i)} \cdots \beta_{n}^{(i)}$, where $\alpha_{k}^{(i)}$ and $\beta_{k}^{(i)}$ are in $\Sigma$ for $1 \leq k \leq n$.

Let

$$
\operatorname{gain}(S, k, z)=E\left(S, z_{1} \cdots z_{k-1} z \pi_{k+1} \cdots \pi_{n}\right)-E\left(S, z_{1} \cdots z_{k-1} \pi_{k} \pi_{k+1} \cdots \pi_{n}\right)
$$

for $z \in \Omega_{1}$ and $1 \leq k \leq n$. For the proof, it suffices to prove that for each $1 \leq k \leq n$ there is some $z$ in $\Omega_{1}$ such that gain $(S, k, z) \geq 0$. In order to describe $P\left(\left(\alpha^{(i)}, \beta^{(i)}\right), z_{1} \cdots z_{k-1} \pi_{k} \cdots \pi_{n}\right)$ conveniently, let

$$
\begin{aligned}
A_{k}^{(i)} & =\prod_{j \in\left\{j \geq k \mid \alpha_{j}^{(i)}=\beta_{j}^{(i)}\right\}} p_{j}\left(S_{\alpha_{j}^{(i)}}\right), \\
B_{k}^{(i)} & =\prod_{j \in\left\{j \geq k \mid \alpha_{j}^{(i)} \neq \beta_{j}^{(i)}\right\}} p_{j}\left(S_{\alpha_{j}^{(i)}}\right), \\
C_{k}^{(i)} & =\prod_{j \in\left\{j \geq k \mid \alpha_{j}^{(i)} \neq \beta_{j}^{(i)}\right\}} p_{j}\left(S_{\alpha_{j}^{(i)}} \cap S_{\beta_{j}^{(i)}}\right) .
\end{aligned}
$$


We first classify the set $I=\{1, \ldots, m\}$ of indices into the following three sets:

$$
\begin{aligned}
& I_{1}=\left\{i \mid \forall j_{1}\left(1 \leq j_{1}<k\right)\left[\alpha_{j_{1}}^{(i)}, \beta_{j_{1}}^{(i)} \in z_{j_{1}}\right]\right\} \\
& I_{2}=\left\{i \mid\left(\forall j_{1}\left(1 \leq j_{1}<k\right)\left[\alpha_{j_{1}}^{(i)} \in z_{j_{1}}\right]\right) \wedge\left(\exists j_{2}\left(1 \leq j_{2}<k\right)\left[\beta_{j_{2}}^{(i)} \notin z_{j_{2}}\right]\right)\right\} \\
& I_{3}=\left\{i \mid \exists j_{1}\left(1 \leq j_{1}<k\right)\left[\alpha_{j_{1}}^{(i)} \notin z_{j_{1}}\right]\right\}
\end{aligned}
$$

Then

$$
P\left(\left(\alpha^{(i)}, \beta^{(i)}\right), z_{1} \cdots z_{k-1} \pi_{k} \cdots \pi_{n}\right)= \begin{cases}A_{k}^{(i)} \cdot\left(B_{k}^{(i)}-C_{k}^{(i)}\right) & \text { if } i \in I_{1}, \\ A_{k}^{(i)} \cdot B_{k}^{(i)} & \text { if } i \in I_{2}, \\ 0 & \text { if } i \in I_{3} .\end{cases}
$$

By an easy calculation, for $z$ in $\Omega_{1}$ we have

$$
\begin{aligned}
\operatorname{gain}(S, k, z)= & \sum_{\substack{i \in I_{1} \\
\alpha_{k}^{(i)}=\beta_{k}^{(i)} \\
\alpha_{k}^{(i)} \in z}}\left(1-p_{k}\left(S_{\alpha_{k}^{(i)}}\right)\right) \cdot A_{k+1}^{(i)} \cdot\left(B_{k+1}^{(i)}-C_{k+1}^{(i)}\right) \\
+ & \sum_{\substack{i \in I_{1} \\
\alpha_{k}^{(i)} \neq \beta_{k}^{(i)} \\
\alpha_{k}^{(i)}, \beta_{k}^{(i)} \in z}}^{(i)} A_{k+1}^{(i)} \cdot\left(\left(1-p_{k}\left(S_{\alpha_{k}^{(i)}}\right)\right) \cdot B_{k+1}^{(i)}-\left(1-p_{k}\left(S_{\alpha_{k}^{(i)}} \cap S_{\beta_{k}^{(i)}}\right)\right) \cdot C_{k+1}^{(i)}\right) \\
+ & \sum_{\substack{i \in I_{1} \\
\alpha_{k}^{(i)} \neq \beta_{k}^{(i)} \\
\alpha_{k}^{(i)} \in z, \beta_{k}^{(i)} \notin z}} A_{k+1}^{(i)} \cdot\left(\left(1-p_{k}\left(S_{\alpha_{k}^{(i)}}\right)\right) \cdot B_{k+1}^{(i)}+C_{k}^{(i)}\right) \\
- & \sum_{\substack{i \in I_{1} \\
\alpha_{k}^{(i)} \notin z}} A_{k}^{(i)} \cdot\left(B_{k}^{(i)}-C_{k}^{(i)}\right) \\
+ & \sum_{\substack{i \in I_{2} \\
\alpha_{k}^{(i)} \in z}}\left(1-p_{k}\left(S_{\left.\alpha_{k}^{(i)}\right)}\right) \cdot A_{k+1}^{(i)} \cdot B_{k+1}^{(i)}\right. \\
- & \sum_{\substack{i \in I_{2} \\
\alpha_{k}^{(i)} \notin z}} A_{k}^{(i)} \cdot B_{k}^{(i)} .
\end{aligned}
$$

We shall show that

$$
\sum_{z \subseteq \Sigma}|z| \cdot \operatorname{gain}(S, k, z) \geq 0
$$

if $p_{1}, \ldots, p_{n}$ satisfy the conditions of the theorem. Then this implies that gain $(S, k, z) \geq 0$ for some $z$ in $\Omega_{1}$.

We evaluate the above sum in the six parts (1)-(6) constituting gain $(S, k, z)$.

Part (1): Let $D(i, k)=\left(1-p_{k}\left(S_{\alpha_{k}^{(i)}}\right)\right) \cdot A_{k+1}^{(i)} \cdot\left(B_{k+1}^{(i)}-C_{k+1}^{(i)}\right)$.

$$
\begin{aligned}
\sum_{r=1}^{s} r \sum_{\substack{z \subseteq \Sigma \\
|z|=r}} \sum_{\substack{i \in I_{1} \\
\alpha_{k}^{(i)}=\beta_{k}^{(i)} \\
\alpha_{k}^{(i)} \in z}} D(i, k) & =\sum_{r=1}^{s} r \sum_{\sigma \in \Sigma} \sum_{\substack{z \subseteq \Sigma \\
|z|=r}} \sum_{\substack{i \in I_{1} \\
\alpha_{k}^{(i)}=\beta_{k}^{(i)} \\
\alpha_{k}^{(i)}=\sigma \in z}} D(i, k)=\sum_{r=1}^{s} r\left(\begin{array}{c}
s-1 \\
r-1
\end{array}\right) \sum_{\substack{i \in I_{1} \\
\alpha_{k}^{(i)}=\beta_{k}^{(i)}}} D(i, k) \\
& =(s+1) \cdot 2^{s-2} \sum_{\substack{i \in I_{1} \\
\alpha_{k}^{(i)}=\beta_{k}^{(i)}}} D(i, k)
\end{aligned}
$$

Part (2): Let $E(i, k)=A_{k+1}^{(i)} \cdot\left(\left(1-p_{k}\left(S_{\alpha_{k}^{(i)}}\right)\right) \cdot B_{k+1}^{(i)}-\left(1-p_{k}\left(S_{\alpha_{k}^{(i)}} \cap S_{\beta_{k}^{(i)}}\right)\right) \cdot C_{k+1}^{(i)}\right)$.

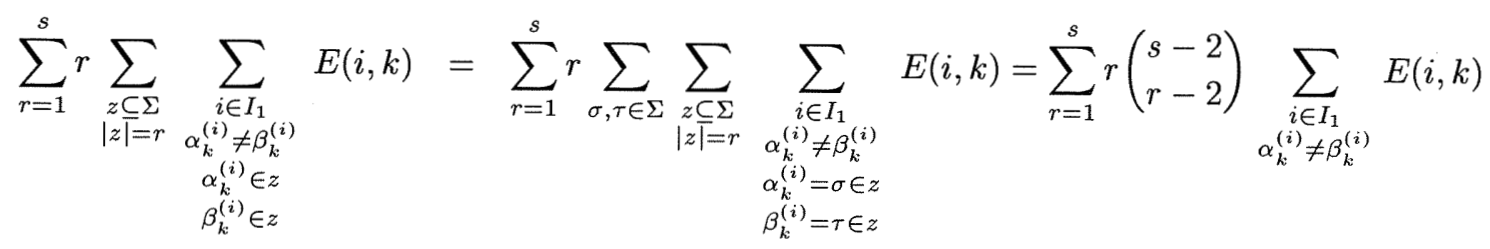




$$
=(s+2) \cdot 2^{s-3} \sum_{\substack{i \in I_{1} \\ \alpha_{k}^{(i)} \neq \beta_{k}^{(i)}}} E(i, k)
$$

Part (3): Let $F(i, k)=A_{k+1}^{(i)} \cdot\left(\left(1-p_{k}\left(S_{\alpha_{k}^{(i)}}\right)\right) \cdot B_{k+1}^{(i)}+C_{k}^{(i)}\right)$.

$$
\begin{aligned}
\sum_{r=1}^{s} r \sum_{\substack{z \subseteq \Sigma \\
|z|=r}} \sum_{\substack{i \in I_{1} \\
\alpha_{k}^{(i)} \neq \beta_{k}^{(i)} \\
\alpha_{k}^{(i)} \in z \\
\beta_{k}^{(i)} \notin z}} F(i, k) & =\sum_{r=1}^{s} r \sum_{\sigma, \tau \in \Sigma} \sum_{\substack{z \subseteq \Sigma \\
|z|=r}} \sum_{\begin{array}{c}
i \in I_{1} \\
\alpha_{k}^{(i)} \neq \beta_{k}^{(i)} \\
\alpha_{k}^{(i)}=\sigma \in z \\
\beta_{k}^{(i)} \notin z
\end{array}} F(i, k)=\sum_{r=1}^{s} r\left(\begin{array}{c}
s-2 \\
r-1
\end{array}\right) \sum_{\substack{i \in I_{1} \\
\alpha_{k}^{(i)} \neq \beta_{k}^{(i)}}} F(i, k) \\
& =s \cdot 2^{s-3} \sum_{\substack{i \in I_{1} \\
\alpha_{k}^{(i)} \neq \beta_{k}^{(i)}}} F(i, k)
\end{aligned}
$$

Part (4): Let $G(i, k)=A_{k}^{(i)} \cdot\left(B_{k}^{(i)}-C_{k}^{(i)}\right)$.

$$
\begin{aligned}
\sum_{r=1}^{s} r \sum_{\substack{z \subseteq \Sigma \\
|z|=r}} \sum_{\substack{i \in I_{1} \\
\alpha_{k}^{(i)} \notin z}} G(i, k) & =\sum_{r=1}^{s} r \sum_{\sigma \in \Sigma} \sum_{\substack{z \subseteq \Sigma \\
|z|=r}} \sum_{\substack{i \in I_{1} \\
\alpha_{k}^{(i)}=\sigma \notin z}} G(i, k)=\sum_{r=1}^{s} r\left(\begin{array}{c}
s-1 \\
r
\end{array}\right) \sum_{i \in I_{1}} G(i, k) \\
& =(s-1) \cdot 2^{s-2} \sum_{i \in I_{1}} G(i, k)
\end{aligned}
$$

(5) and (6) are similar to Part (1) and (4), respectively.

Part (5): Let $H(i, k)=\left(1-p_{k}\left(S_{\alpha_{k}^{(i)}}\right)\right) \cdot A_{k+1}^{(i)} \cdot B_{k+1}^{(i)}$.

$$
\sum_{r=1}^{s} r \sum_{\substack{z \subseteq \Sigma \\
|z|=r}} \sum_{\substack{i \in I_{2} \\
\alpha_{k}^{(i)} \in z}} H(i, k)=\sum_{r=1}^{s} r\left(\begin{array}{c}
s-1 \\
r-1
\end{array}\right) \sum_{i \in I_{2}} H(i, k)=(s+1) \cdot 2^{s-2} \sum_{i \in I_{2}} H(i, k)
$$

Part (6): Let $I(i, k)=A_{k}^{(i)} \cdot B_{k}^{(i)}$.

$$
\sum_{r=1}^{s} r \sum_{\substack{z \subseteq \Sigma \\
|z|=r}} \sum_{\substack{i \in I_{2} \\
\alpha_{k}^{(i)} \notin z}} H(i, k)=\sum_{r=1}^{s} r\left(\begin{array}{c}
s-1 \\
r
\end{array}\right) \sum_{i \in I_{2}} H(i, k)=(s-1) \cdot 2^{s-2} \sum_{i \in I_{2}} H(i, k)
$$

By definition, if $\alpha_{k}^{(i)}=\beta_{k}^{(i)}$, then $A_{k}^{(i)}=p_{k}\left(S_{\alpha_{k}^{(i)}}\right) \cdot A_{k+1}^{(i)}, B_{k}^{(i)}=B_{k+1}^{(i)}$, and $C_{k}^{(i)}=C_{k+1}^{(i)}$. If $\alpha_{k}^{(i)} \neq \beta_{k}^{(i)}$, then $A_{k}^{(i)}=A_{k+1}^{(i)}, B_{k}^{(i)}=p_{k}\left(S_{\alpha_{k}^{(i)}}\right) \cdot B_{k+1}^{(i)}$, and $C_{k}^{(i)}=p_{k}\left(S_{\alpha_{k}^{(i)}} \cap S_{\beta_{k}^{(i)}}\right) \cdot C_{k+1}^{(i)}$. Then, by using these relations, we can show the following equation:

$$
\begin{aligned}
\sum_{z \subseteq \Sigma}|z| \cdot \operatorname{gain}(S, k, z) & =(7)+(8)+(9)-(10)+(11)-(12) \\
& =\sum_{\substack{i \in I_{1} \\
\alpha_{k}^{(i)}=\beta_{k}^{(i)}}}\left((s+1) \cdot 2^{s-2}-s \cdot 2^{s-1} \cdot p_{k}\left(S_{\alpha_{k}^{(i)}}\right)\right) \cdot A_{k+1}^{(i)} \cdot\left(B_{k+1}^{(i)}-C_{k+1}^{(i)}\right) \\
& +\sum_{\substack{i \in I_{1} \\
\alpha_{k}^{(i)} \neq \beta_{k}^{(i)}}}\left((s+1) \cdot 2^{s-2}-s \cdot 2^{s-1} \cdot p_{k}\left(S_{\alpha_{k}^{(i)}}\right)\right) \cdot A_{k+1}^{(i)} \cdot B_{k+1}^{(i)} \\
& +\sum_{\substack{i \in I_{1} \\
\alpha_{k}^{(i)} \neq \beta_{k}^{(i)}}}\left(s \cdot 2^{s-1} \cdot p_{k}\left(S_{\alpha_{k}^{(i)}} \cap S_{\beta_{k}^{(i)}}\right)-(s+2) \cdot 2^{s-3}\right) \cdot A_{k+1}^{(i)} \cdot C_{k+1}^{(i)} \\
& +\sum_{i \in I_{2}}\left((s+1) \cdot 2^{s-2}-s \cdot 2^{s-1} \cdot p_{k}\left(S_{\alpha_{k}^{(i)}}\right)\right) \cdot A_{k+1}^{(i)} \cdot B_{k+1}^{(i)} .
\end{aligned}
$$

Therefore, if $p_{k}$ satisfies $p_{k}\left(S_{\sigma}\right) \leq \frac{s+1}{2 s}$ for all $\sigma$ in $\Sigma$ and $p_{k}\left(S_{\sigma} \cap S_{\tau}\right) \geq \frac{s+2}{4 s}$ for all $\sigma, \tau$ in $\Sigma$, then $\sum_{z \subseteq \Sigma}|z|$. $\operatorname{gain}(S, k, z) \geq 0$.

Theorem 2 has an advantage that it allows variations for motifs by specifying the probabilities for $z$ in $\Omega_{1}$ as long as they satisfy the two conditions in Theorem 2. As a corollary of Theorem 2, we can prove the following lower bounds of the expectation: 
Corollary 1 Let $m=|S|, s=|\Sigma|$ and let $n$ be the length of a motif.

(1) If $p_{k}(Z)=\frac{|Z|}{s \cdot 2^{s-1}}$ for all $Z$ in $\Omega_{1}$ and for all $1 \leq k \leq n$, then

$$
E\left(S, \pi_{1} \cdots \pi_{n}\right) \geq \frac{m}{4} \cdot\left(\frac{s+1}{2 s}\right)^{n-1}
$$

This is the case that any $z \in \Omega_{1}$ is allowed for a motif.

(2) If $p_{k}(\{\sigma\})=\frac{1}{2 s}$ for all $\sigma$ in $\Sigma, p_{k}(\Sigma)=\frac{1}{2}$ and $p_{k}(Z)=0$ for other $z$ in $\Omega_{1}$ for all $1 \leq k \leq n$, then

$$
E\left(S, \pi_{1} \cdots \pi_{n}\right) \geq \frac{m}{2 s} \cdot\left(\frac{s+1}{2 s}\right)^{n-1} .
$$

This is the case that only $\Sigma$ and $\{\sigma\}$ for $\sigma \in \Sigma$ are allowed for a motif.

From Theorem 2, the greedy algorithm in Fig. 3 produces a motif $\hat{\pi}=z_{1} \cdots z_{n}$ with $\operatorname{cost}(S, \hat{\pi})$ at least as large as $E\left(S, \pi_{1} \cdots \pi_{n}\right)$. The lower bounds of $E\left(S, \pi_{1} \cdots \pi_{n}\right)$ in Corollary 1 are not good when $n$ and $s$ are larger. In [13], we have implemented this greedy algorithm and applied to finding motifs for exon/intron splicing sites and E. coli promoters. The experimental results in [13] reveal that this algorithm can achieve much better performance in practice.

It is yet another method to apply the approximation algorithm for the maximum generalized satisfiability problem (MAXGSAT) (see Theorem 13. 2 in [8]) by reformulating the problem as MAXGSAT. Given a set $\Psi=\left\{\psi_{1}, \ldots, \psi_{m}\right\}$ of boolean expressions in $n$ variables, this approximation algorithm produces a truth assignment satisfying at least $\frac{m}{2^{k}}$ expressions in $\Psi$, where $k$ is the maximum number of distinct variables in $\psi_{i}$ for $1 \leq i \leq m$. For this purpose, we transform a yes-no sample $S=\left\{\left(\alpha^{(1)}, \beta^{(1)}\right), \ldots,\left(\alpha^{(m)}, \beta^{(m)}\right)\right\}$ to a collection $\Psi=\left\{\psi_{1}, \ldots, \psi_{m}\right\}$ of boolean expressions as follows: Let $s=|\Sigma|$. We use boolean variables $\pi_{k}[z]$ for $1 \leq k \leq n$ and $z \in \Omega_{1}$ in a way that $\pi_{k}[z]=$ true $\Leftrightarrow \pi_{k}=z$. Let

$$
\varphi=\bigwedge_{k=1}^{n}\left(\left(\bigvee_{z \in \Omega_{1}} \pi_{k}[z]\right) \wedge\left(\bigwedge_{z \neq z^{\prime} \in \Omega_{1}}\left(\overline{\pi_{k}}[z]+\overline{\pi_{k}}\left[z^{\prime}\right]\right)\right)\right) .
$$

Obviously, a truth assignment satisfying $\mu$ determines a motif $\pi=z_{1} \cdots z_{n}$. Then for $\left(\alpha^{(i)}, \beta^{(i)}\right)$, let $\psi_{i}$ be a boolean expression with these $n \cdot\left(2^{s}-1\right)$ variables defined by

$$
\psi_{i}=\varphi \wedge\left(\left(\bigwedge_{k=1}^{n} \bigvee_{\alpha_{k}^{(i)} \in z \in \Omega_{1}} \pi_{k}[z]\right) \wedge\left(\bigvee_{k=1}^{n} \bigvee_{\beta_{k}^{(i)} \in z \in \Omega_{1}} \overline{\pi_{k}}[z]\right)\right)
$$

Let $\pi=z_{1} \cdots z_{n}$ be a motif determined by a truth assignment. Then $\psi_{i}$ is satisfied if and only if $\left(\alpha^{(i)}, \beta^{(i)}\right)$ is good for $\pi$ for each $1 \leq i \leq m$. By applying the approximation algorithm for MAXGSAT to the instance $\Psi=\left\{\psi_{1}, \ldots, \bar{\psi}_{m}\right\}$, we obtain a motif $\pi$ with

$$
\operatorname{cost}(S, \pi) \geq \frac{m}{2^{n \cdot\left(2^{s}-1\right)}}
$$

This lower bound becomes drastically worse if the size $s$ of $\Sigma$ gets larger.

\section{Conclusion}

First, we defined the best consensus motif problem (BCM) motivated by the problem of extracting motifs from positive and negative sequences. Second, we proved the NP-completeness of the BCM problem for type $\Omega_{1}=\{z \mid \emptyset \neq z \subseteq \Sigma\}$. Furthermore, for this problem, we devised a polynomial-time greedy algorithm and showed a lower bound of its performance. This problem has a special importance when data are provided as tuples of values of attributes $A_{1}, \ldots, A_{n}$ 
as in [9]. We do not know whether there is any polynomial time algorithm with better performance. We also showed that the BCM problem for type $\Omega_{\infty}=\Omega_{1} \cup\left\{\Sigma^{+}\right\} \cup\left\{\Sigma^{[i, j]} \mid 1 \leq i \leq j\right\}$ is NP-complete. From both theory and practice, it is an challenging open problem to devise any efficient approximation algorithm for finding such general motifs together with a good performance guarantee.

Aiming at knowledge discovery from amino acid sequences, the third author's research group has developed a system called BONSAI Garden [2, 10, 12] that produces, from positive and negative examples, a mapping $\psi$ called an alphabet indexing which classifies twenty amino acid residues into a smaller categories and a decision tree whose internal nodes are labeled with regular patterns. Since regular patterns are used for making decisions at nodes, only exact pattern matching is allowed. We are planing to implement the algorithm developed in this paper in a forthcoming version of BONSAI Garden so that it can cope with sequences with ambiguity.

\section{Acknowledgments}

The authors would like to thank Satoru Kuhara for his guidance in Genome Informatics. This work is partly supported by Grant-in-Aid for Scientific Research on Priority Area "Genome Informatics", No. 06558047 and No. 06680326 from Ministry of Education, Science and Culture, Japan.

\section{References}

[1] Angluin, D., Finding patterns common to a set of strings, J. Comput. System Sci. 21 (1980) $46-62$.

[2] Arikawa, S., Miyano, S., Shinohara, A., Kuhara, S., Mukouchi, Y., and Shinohara, T., A machine discovery from amino acid sequences by decision trees over regular patterns, New Generation Computing 11 (1993) 361-375.

[3] Bairoch, A., PROSITE: a dictionary of sites and patterns in proteins, Nucleic Acids Res. 19 (1991) 2241-2245.

[4] Garey, M.R., Johnson, D.S. and Stockmeyer, L., Some simplified NP-complete problems, Theoret. Comput. Sci. 1 (1976) 237-267.

[5] Gribskov, M. and Devereux, J., Sequence Analysis Primer, Stockholm Press, 1991.

[6] Jiang, T. and Li, M., On the complexity of learning strings and sequences, Proc. 4th Workshop on Computational Learning Theory, 1991, 367-371.

[7] Miyano, S., Shinohara, A. and Shinohara, T., Which classes of elementary formal systems are polynomial-time learnable?, Proc. Second Workshop on Algorithmic Learning Theory, 1991, 139150.

[8] Papadimitriou, C.H., Computational Complexity, Addison-Wesley, 1994.

[9] Quinlan, J.R., Induction on decision trees, Machine Learning 1 (1986) 81-106.

[10] Shimozono, S., Shinohara, A., Shinohara, T., Miyano, S., Kuhara, S., and Arikawa, S., Knowledge acquisition from amino acid sequences by machine learning system BONSAI, Transactions of Information Processing Society of Japan 35 (1994) 2009-2018.

[11] Shinohara, T., Polynomial time inference of extended regular pattern languages, Lecture Notes in Computer Science 147 (1983) 115-127.

[12] Shoudai, T., Lappe, M., Miyano, S., Shinohara, A., Okazaki, T., Arikawa, S., Uchida, T., Shimozono, S., Shinohara, T., and Kuhara, S., BONSAI Garden: parallel knowledge discovery system for amino acid sequences, Proc. Third International Conference on Intelligent Systems for Molecular Biology (AAAI Press), 1995, 359-366.

[13] Tateishi, E. and Miyano, S., A greedy strategy for finding motifs from positive and negative examples, RIFIS-TR-CS-118, Research Institute of Fundamental Information Science, Kyushu University, 1995.

[14] Yannakakis, M., On the approximation of maximum satisfiability, J. Algorithms 17 (1994) 475502. 